\title{
DGLAP Evolution and Saturation Effects at HERA
}

\author{
Henri Kowalski* \\ Deutsches Elektronen Synchrotron DESY, Hamburg Germany \\ E-mail: 'Henri.Kowalski@desy.dè
}

Abstract: We discuss HERA data on the high energy behavior of the total $\gamma^{*} p$ cross section and on diffraction in DIS. A natural link between inclusive and diffractive $\gamma^{*} p$ processes is provided by the dipole description of DIS. In the dipole picture the hard and soft interaction regions can be connected by the saturation hypothesis which leads to a good description of HERA measurements. An extension of the saturation ansatz by the evolution effects improves sizably the agreement with data.

\section{The total and diffractive $\gamma^{*} p$ cross section}

Let us first recall that at HERA the electrons collide with protons by emitting highly energetic photons which then hit the proton. Hence, by studying electron-proton collisions, in fact, we investigate photon-proton scattering processes at high energies (in short: $\gamma^{*} p$ ) where the photon is highly virtual. The energies of these photons, in the proton rest system, can go up to $50000 \mathrm{GeV}$.

One of the most important measurements at HERA is the determination of the total $\gamma^{*} p$ cross section as a function of the photon virtuality, $Q^{2}$, and of the energy of the

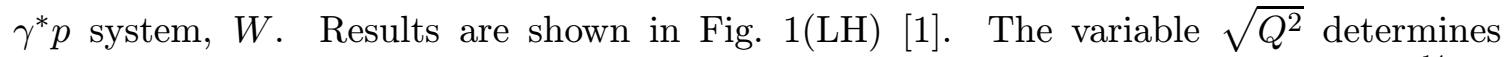
the transverse distance inside the proton that the photon can "resolve", $d \approx \frac{2 \cdot 10^{-14} \mathrm{~cm}}{Q(\mathrm{GeV})}$. Beginning at the top of our plot with small $Q^{2}$-values (large transverse sizes) the photon resembles a hadron, e.g. a $\rho$-meson. As the $Q^{2}$-values increase the photon shrinks and becomes more and more point-like. Following the rise of the total cross section in $W$ as a function of $Q^{2}$, we observe a striking change. At small $Q^{2}$ the behavior is still very much the same as in hadron-hadron scattering. With increasing $Q^{2}$, the rise in $W$ becomes stronger (at the same time the overall magnitude of the cross section decreases). If we parameterize the $W$-dependence by a power law, $\sigma_{\text {tot }}^{\gamma^{*} p} \sim\left(W^{2}\right)^{\lambda_{t o t}}$, this behavior is translated into the $Q^{2}$ dependence of the exponent $\lambda_{t o t}$, Fig. i i. the hadronic value $\lambda_{t o t}=0.08$, whereas for larger values of $Q^{2}$ the exponent $\lambda_{t o t}$ increases

${ }^{*}$ Speaker. 

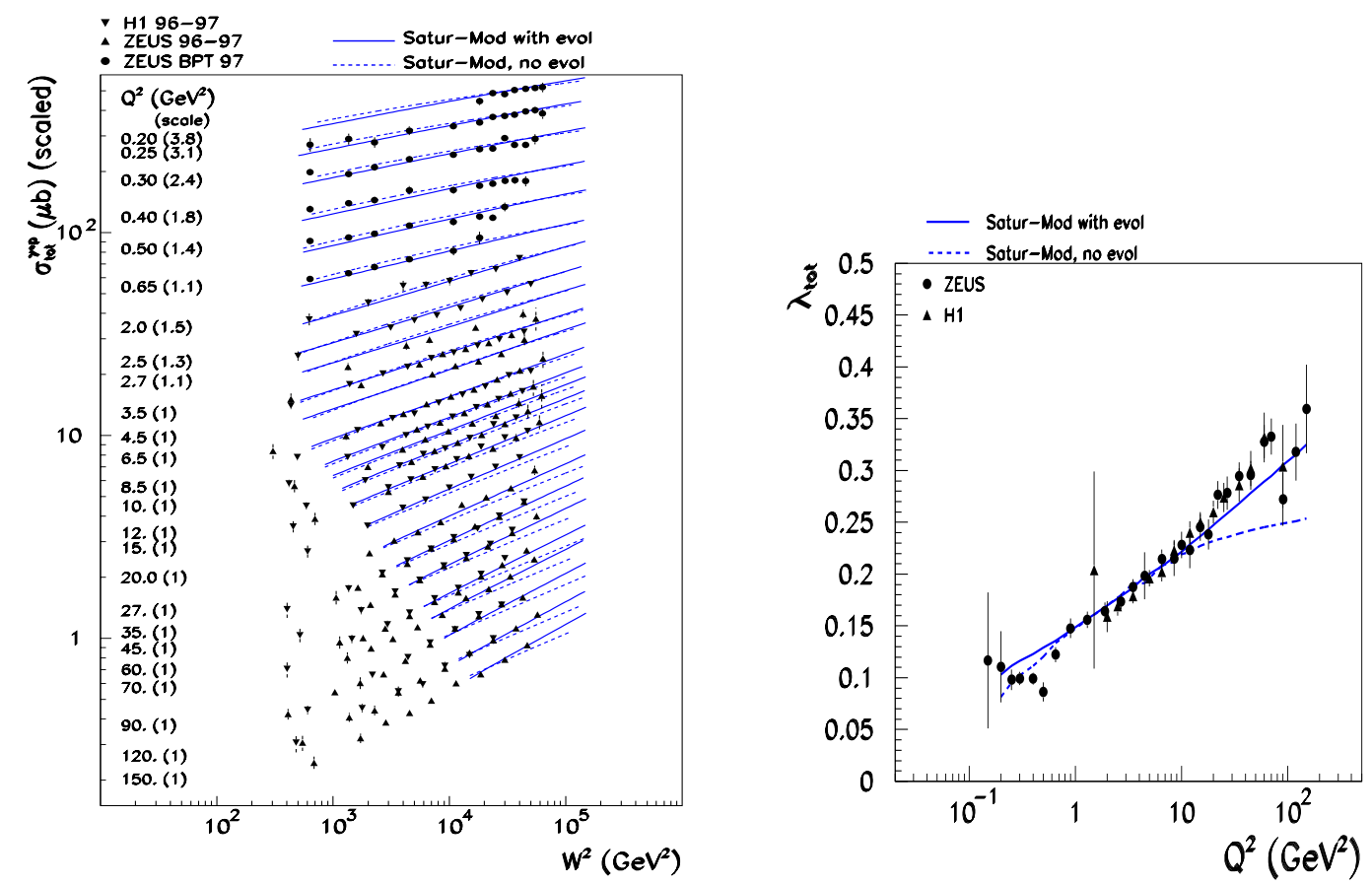

Figure 1: $(L H) \gamma^{*} p$ cross section as a function of $W^{2}$ at various $Q^{2}$. The values of $Q^{2}$ are shown on the left side together with the scale factor applied to the data for a better visibility. The full line shows a fit by a saturation model with evolution $[\overline{2}]$, the dashed line shows a fit by the original model $[\overline{3}]$. The fits are performed for $x<0.01$. (RH) The exponent $\lambda_{t o t}$ in the parameterization $\sigma_{\text {tot }}^{\gamma^{*} p} \sim\left(W^{2}\right)^{\lambda_{\text {tot }}}$, plotted as a function of $Q^{2}$.

substantially. In this plot the l.h.s. belongs to the hadronic world, the r.h.s. to the smalldistance world where pQCD is expected to apply. QCD calculations allow the results of the small-distance part (large $Q^{2}$ values) to be interpreted in terms of QCD radiation [4]-

Another important observation of HERA is the measurement of the diffractive $\gamma^{*} p$ cross section. Before HERA it was not expected that diffractive final states in deep inelastic scattering would play a significant role: the energetic virtual photon smashes the proton into pieces, the final state contains large numbers of particles or jets, and it was expected that the $q \bar{q}$-pair created by the photon could hardly be separated from the rest of the final state. In contrast to this expectation, a sizable and approximately constant fraction of diffractive events was observed as a function of $W$, Fig. $\underset{2}{2}(\mathrm{LH})$.

\section{Dipole description of DIS}

The observation of diffractive processes at HERA leads to a dipole picture of DIS. In the dipole description the probability for the incoming virtual photon to fluctuate into quarkantiquark pair is given by the transversely $(t)$ and longitudinally $(l)$ polarized photon wave-functions $\psi\left(Q^{2}, z, \vec{r}\right)_{t, l}$ ( $z$ denotes here the momentum fraction of the photon carried by the quark and $\vec{r}$ the transverse size of the quark-antiquark pair). The wave functions 

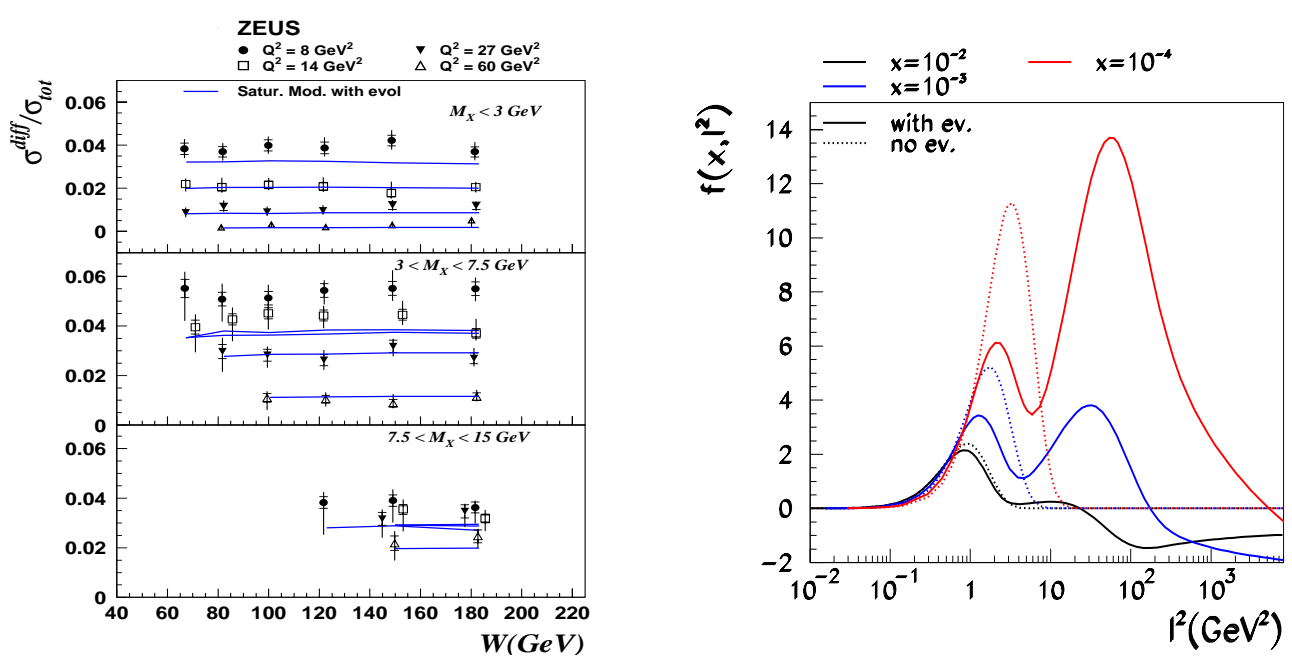

Figure 2: (LH) Ratio of diffractive and total cross sections at fixed values of $Q^{2}$, for different regions of the invariant diffractive mass $M_{X}$. The lines belong to a model [2] described in the text. (RH) Comparisons of unintegrated gluon distributions $f\left(x, l^{2}\right)$ with (full line) and without (dotted line) evolution effects for different $x$ values.

are solely determined by the coupling of the photon to the quark pair, and in the present computations they are mostly assumed to be the same as in QED. The emission of the gluon cloud and its interaction with the proton is described by the dipole cross section, $\sigma_{q \bar{q} p}(x, \vec{r})$, which is closely related to the (unintegrated) gluon distribution (see below). All interesting dynamics is encoded in the $\vec{r}$ and $x$ dependence of $\sigma_{q \bar{q} p}$.

In the proton rest frame the total cross section can be written in the following form:

$$
\sigma_{t, l}^{\gamma^{*} p}\left(x, Q^{2}\right)=\int d^{2} \vec{r} \int d z \psi\left(Q^{2}, z, \vec{r}\right)_{t, l}^{*} \sigma_{q \bar{q} p}(x, \vec{r}) \psi\left(Q^{2}, z, \vec{r}\right)_{t, l}
$$

The Optical Theorem applied in the framework of the dipole picture allows to express the cross section of diffractive $q \bar{q}$ production in which proton remains intact as:

$$
\left.\frac{d \sigma_{D i f f}^{\gamma^{*} p}}{d t}\right|_{t=0}=\frac{1}{16 \pi} \int d^{2} \vec{r} \int d z \psi\left(Q^{2}, z, \vec{r}\right)^{*} \sigma_{q \bar{q} p}^{2}\left(x, r^{2}\right) \psi\left(Q^{2}, z, \vec{r}\right)
$$

The total and diffractive cross sections are expressed by the same elements. This shows the general property that the structure functions related to these cross sections, $F_{2}$ and $F_{2}^{D(4)}$, are of the same fundamental importance. Hence simultaneous observation of the inclusive and diffractive DIS processes allows us to get a better insight into the proton structure.

The equations $\overline{2} \cdot \bar{l}_{1}^{1}$ and $\overline{2} . \overline{2}$ s should allow direct determination of $\sigma_{q \bar{q} p}$, i.e. the gluon structure of the proton, from data. Today, however, the precision of the data is not sufficient to invert these equations in a straightforward way. In order to describe the data it is necessary to make specific assumptions about the dipole cross section. A good description of the HERA data has recently been obtained by a model built on the idea of saturation. 


\section{Saturation Model}

The idea of saturation [i-i] emerges from the study of the rise of the total $\gamma^{*} p$ cross section with energy. This rise can be explained in QCD by the emission of a single gluon cascade. Since the increase of the total $\gamma^{*} p$ cross section with energy cannot continue forever, there must be some corrections which slow down the rise. The one-cascade picture can be valid only if the system of gluons is sufficiently dilute. When at very small $x$ the gluon density of the single cascade becomes too high, interactions with other cascades will become important. This process, called saturation, leads to a damping of the growth of cross sections with energy. It can be modeled by a simple ansatz for the dipole cross section [i]in:

$$
\sigma_{q \bar{q} p}(x, \vec{r})=\sigma_{0}\left(1-\exp \left[-\frac{r^{2}}{4 R_{0}^{2}}\right]\right)
$$

where $R_{0}$ denotes the (x-dependent) dipole size where saturation sets in ("saturation radius": $R_{0}^{2}=R_{0}^{2}(x)=\frac{1}{Q_{0}^{2}}\left(\frac{x}{x_{0}}\right)^{\lambda_{G B W}}$ with $\left.Q_{0}=1 \mathrm{GeV}\right)$. The parameters of the model, $\sigma_{0}$, $x_{0}$, and $\lambda_{G B W}$, were determined from the fit to the total cross section data.

The model describes the damping of the rise of the cross sections at high energies for higher $Q^{2}$ (outside of the energy range accessible to HERA) as an effect of the diminishing saturation radius. At low $Q^{2}$ the dipole sizes are large compared to the saturation radius which leads to the typical hadronic rate of rise, $\lambda_{t o t}=0.08$. The dashed curves in Fig. Iili show that the energy dependence of the total cross section is well described by the original model for $Q^{2}$ region up to around $20 \mathrm{GeV}^{2}$.

The model has also been used for diffraction: inserting the formula for the dipole cross section into eq. ( $\overline{2} . \overline{2}$, $)$, a good description of the diffractive cross section for $q \bar{q}$ production is obtained. In particular, as a result of a subtle interplay between the photon wave function and the dipole cross section, it succeeds in reproducing the energy dependence shown in Fig. $\overline{2}_{1}^{\prime}(\mathrm{LH})$.

\section{DGLAP evolution in the dipole models}

The unintegrated gluon distribution gives the ordinary gluon distribution $g\left(x, Q^{2}\right)$ through

$$
x g\left(x, Q^{2}\right)=\int_{0}^{Q^{2}} \frac{d l^{2}}{l^{2}} f\left(x, l^{2}\right)
$$

provided we have a model for $f\left(x, l^{2}\right)$ in the nonperturbative region of small $l^{2}$. The unintegrated gluon distribution can can be expressed by the dipole cross section as

$$
\frac{\alpha_{s} f\left(x, l^{2}\right)}{l^{4}}=\frac{3}{4 \pi} \int \frac{d^{2} \mathbf{r}}{(2 \pi)^{2}} \exp \{i \mathbf{l} \cdot \mathbf{r}\}\left\{\sigma_{0}-\sigma_{q \bar{q} p}(x, r)\right\}
$$

For small enough $r$ we find

$$
\hat{\sigma}(x, \mathbf{r}) \simeq \frac{\pi^{2}}{3} r^{2} \alpha_{s} x g\left(x, C / r^{2}\right)
$$


Expanding the exponential in the saturation model ( $\overline{3} . \overline{1})$, for $r \ll 2 R_{0}(x)$, and by comparing with expression (4) we find the following gluon distribution

$$
x g\left(x, Q^{2}\right)=\frac{3}{4 \pi^{2} \alpha_{s}} \frac{\sigma_{0}}{R_{0}^{2}(x)}
$$

which is independent of $Q^{2}$. This is in contradiction to the fact that, according to the DGLAP evolution equations, the ordinary gluon distribution depends logarithmically on $Q^{2}$. Thus we need to modify the saturation model to take into account the DGALP evolution at small values of $r$. The physics of saturation however, which reflects unitarity, should not be altered.

Therefore, we propose the following modification of the model $\left(\overline{\overline{3}} \cdot \overline{1}_{1}\right)$

$$
\sigma_{q \bar{q} p}(x, r)=\sigma_{0}\left\{1-\exp \left(-\frac{\pi^{2} r^{2} \alpha_{s}\left(\mu^{2}\right) x g\left(x, \mu^{2}\right)}{3 \sigma_{0}}\right)\right\}
$$

where the scale $\mu^{2}$ is takes the form

$$
\mu^{2}=\frac{C}{r^{2}}+\mu_{0}^{2},
$$

and the parameters $C$ and $\mu_{0}^{2}$ could be determined from best fits to the DIS data. The initial gluon distribution is taken at the initial scale $Q_{0}^{2}=1 \mathrm{GeV}^{2}$ in the form

$$
x g\left(x, Q_{0}^{2}\right)=A_{g} x^{\lambda_{g}}(1-x)^{5.6}
$$

where $A_{g}$ is fitted to the data. Thus, in the present approach, we still have only few parameters, $\sigma_{0}, C, \mu_{0}^{2}, \lambda_{g}$ and/or $A_{g}$ to fit to the data.

The comparison of the data with predictions of the saturation model from Fig. Ii shows a clear improvement of the agreement at larger $Q^{2}$ due to evolution effects. At the same time the important property of the original saturation model, the constant ratio of diffractive and inclusive cross sections as a function of $W$, is preserved, see Fig. 2 i- $(\mathrm{LH})$. The effects of evolution on the unintegrated gluon distribution is shown in Fig. $\operatorname{li}_{2}^{2}(\mathrm{RH})$.

The excellent description of the HERA data presented here shows that saturation and evolution effects can cooperate successfully.

\section{References}

[1] ZEUS Collab. J. Breitweg et al., Phys. Lett. B 487 (2000) 53;

H1 Collab., C. Adloff et al., Eur. Phys. J. C21 (2001) 3;

ZEUS Collab. S. Chekanov et al., Eur. Phys. J. C21 (2001) 443;

[2] J. Bartels, K.Golec-Biernat, H. Kowalski, in preparation

[3] K.Golec-Biernat, M.Wuesthoff, Phys.Rev. D 59 (1999) 014017; Phys.Rev. D 60 (1999) 114023.

[4] J. Bartels, H. Kowalski, Eur. Phys. J. C19 (2001) 693;

[5] ZEUS Collab. J. Breitweg et al., Eur. Phys. J. C1 (1998) 81;

[6] A.H.Mueller "Small-x Physics, High Parton Densities and Parton Saturation in QCD", hep-ph/9911289. 